\title{
Development of autoencoder-based status diagnosis method for ball bearing tribology status monitoring
}

\author{
Ren-Chi Cheng ${ }^{\mathrm{a} 1}{ }^{*}$, Kuo-Shen Chen ${ }^{\mathrm{b} 1}$, Yun-Hui Liu ${ }^{\mathrm{b} 2}$, Lien-Kai Chang ${ }^{\mathrm{c} 1}$, Mi-Ching Tsai ${ }^{\mathrm{b} 1}$ \\ ${ }^{\mathrm{a}}$ Graduate Student, ${ }^{\mathrm{b}}$ Professor, ${ }^{\mathrm{c}}$ Postdoctoral Researcher \\ ${ }^{1}$ Department of Mechanical Engineering, National Cheng-Kung University, Tainan, Taiwan \\ ${ }^{2}$ Department of Mechanical Engineering, Southern Taiwan University of Science and Technology, Tainan, Taiwan \\ *Corresponding Author: n16084292@gs.ncku.edu.tw
}

\begin{abstract}
Ball bearings are widely used in rotating components and definitely influence the operation quality of machines. And their faults are one of the main reasons that make machines break down and this problem should be investigated. Thus, this study develops an effective and flexible diagnosis method for ball bearings. By setting up a rotor bearing-experiment platform and pre-designing failures on bearings or rotating shaft to simulate the service of bearings. Sensors hired are two accelerometers, a microphone, an acoustic emission detector, and a thermal couple. Various statistical methods are used for data reduction and to extract features. Through systematic analysis, it is possible to find the most sensitive features. Those indexes are then fed into autoencorder, which is an unsupervised machine learning scheme, for training the collected data to predict the possible bearing failure type and status. For ease of visualization, the results are mapped into a three-dimensional space for examining the performance in failure diagnosis. The investigation results show that the hired machine learning method performs well with appropriate feature indexes. Finally, specific diagnosis models are created for each corresponding bearing failure condition and a novel whole diagnosis process is proposed to integrate all these models for counting possible multiple causes of failure. This proposed diagnosis flow should be able to significantly improve the prediction accuracy on the reliabilities of rotating machines and could be promoted to other related applications.
\end{abstract}

Keywords: Ball bearing, Rotating machinery, Feature extraction, Machine learning, Autoencoder.

\section{Introduction}

With the expeditious development of technology, the claim of Industry 4.0 has been advocated in order to accelerate productivity, decrease manufacturing cost and improve production quality. Cyber-physical systems, which establish the connection with mechanism and its users on the internet and making users understand the circumstance of it in time, are the key components for realizing the above goals in Industry 4.0 era [1]. For production quality concerns, failure of any component of machines in a factory would impose serious degradation or even break down on whole production lines. Therefore, making the machines in factories have the ability to detect faults of their components by themselves is vital. Nevertheless, it is time consuming and arduous to let the machines detect the faults of all components at the same time without human interventions and machine learning approach seems inevitable for achieving the goal.

Here, a machine learning based diagnosis system for ball bearings is developed and presented in this work as our first step to realize the above-mentioned goal. Bearings can be found virtually in every machine with rotating parts and an accurate status monitoring and reliability evaluation on bearings is therefore extremely important. A bearing may have its particular signatures when the status becomes worse [2]. These signatures could be used as the measure of machine reliability status. To achieve this, one needs to collect signals properly and then process the data to extract features and finally, conduct accurate judgement or estimation based on these features. Accordingly, the problems of bearing diagnosis have been investigated intensively in previous research works. 
Scheffer and Girdhar [2] aggregated some basic dynamic motions of bearing elements. Each movement has a corresponding frequency, such as the fundamental cage frequency, the ball spin frequency, the ball pass inner raceway frequency, and the ball pass outer raceway frequency. In addition to the traditional Fourier transform, many technologies, such as empirical mode decomposition, Hilbert spectrum [3], and continuous wavelet transform [4], are also applied to analyze bearing signals. Furthermore, making good use of diagnosis theories and technologies in actual occasions is an important subject. Randall [5] mounted accelerometers on helicopter gearboxes and successfully separated the signals of bearings and gears to find particular frequencies for detecting incipient failure. Nevertheless, accelerometers may not be the best choice for fault diagnosis. Elasha et al. [6] tried to utilize acoustic emission technology, which was originally developed for static structures, to fault detection of bearings. It implied that using multiple sensors on detection may be worthy of discussing.

With recent rapid advancements in computer science, artificial intelligent (AI) becomes a powerful choice for fault classifications in diagnostic systems. There are various machine learning methods [7] can be applied to automatically diagnostic systems. Li et al. [8] used neural network to classify particular frequencies appeared in bearings. Kankar et al. [9] used statistical methods to extract features for vibration response obtained from various defects of ball bearings to develop faults diagnosis based on artificial neural network (ANN) and support vector machine (SVM). However, the accuracy of ANN and SVM models mentioned was not sufficient. The reason may be attributed to the improper selection of trained features. Thus, conducting sensor index evaluation is required. Furthermore, AI models mentioned above are basically supervised learning methods, in which the trained data must be labeled. There may be a limitation for quantifying severities of failures. On the other hand, unsupervised learning methods are widely used nowadays due to its association rule learning property without labeling. In particular, autoencoder [10], a powerful dimensionality reduction method, is used in many fields, such as image processing [11], cyber security [12], and fault diagnosis [13]. Fig. 1 describes the framework of a basic autoencoder model, which includes encoding and decoding stages. By comparing the error between input and output, the parameters in each layer can be modified. There are several artificial neurons in each layer. The activation function and the number can be decided by the user. Then the dimension of latent space determines the dimension of reduced features. It is flexible for users to change the dimension of encoded features. Moreover, there are many advanced autoencoder algorithm can be selected to improve AI models.

This paper is mainly focused on sensor index evaluation and applying basic autoencoder to develop a diagnosis system for ball bearing failures. Fig. 2 displays the flow chart of overall concept of this study. By setting up an experimental platform with multiple sensors, comprehensive signals of ball bearings can be collected. Then, several time and frequency domain indexes are used to extract features. Sensor index evaluation is conducted subsequently to select appropriate features. Next, autoencoder is used to reduce the dimensions of the features to three dimensions regardless of the number of inputs. Then, plotting the reduced features on three-dimensional coordinates to make it visible and easy to evaluate the effect of trained results. Furthermore, k-means [14], a classification method, is applied to classify the trained results. By comparing the labels given by k-means model and known data inputs, the trained effect can be carried out. The results show that the AI models perform well when the indexes are selected properly. There are mainly three simulation faults of bearings discussing in this research. Each operating condition will have a corresponding diagnosis model. Finally, a whole diagnosis process would be proposed by integrating all models with a particular analyzing flow.

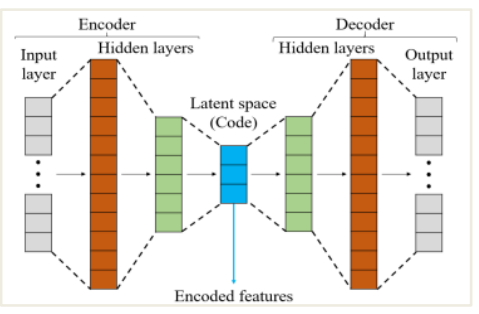

Fig. 1. Procedure of autoencoder.

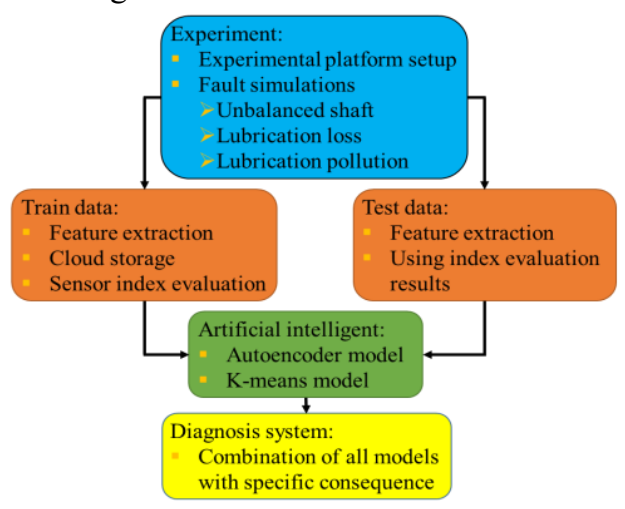

Fig. 2. Flow chart for establishing the diagnosis method. 


\section{Experimental system setup}

\subsection{Experimental platform setup}

In order to obtain the characteristics of the sensor signals in different operating conditions, establishing a flexible, experimental platform is necessary. It is easy and quick to make some faults on the shaft and install the sensors or bearings on the platform.

As shown in Fig. 3(a), a high-speed spindle is put on a tri-axial positioning stage which has an ability to allow the spindle moving along the three directions conveniently. A bearing fixed on a stage composed of steel plates is placed in front of the spindle. The spindle and the bearing are then connected by an aluminum shaft with a diameter of $20 \mathrm{~mm}$. There are two $10 \mathrm{~mm}$ diameter payload disks attached on the shaft. One of them has holes for attaching additional imbalances to induce unbalance of the shaft.

Next, sensors, including two tri-axial accelerometers, a microphone, an acoustic emission detector, and a thermal couple, as well as a current sensor, are installed properly on the testing module to access physical signals of the system. First, one accelerometer is mounted on the outer casing of the spindle and the other is mounted on the casing of the roller bearing. Meanwhile, the microphone is placed nearby the bearing stage. The acoustic emission detector is mounted on the iron plate below the bearing. The thermal couple is attached on the surface of the bearing for accessing the friction heating. Finally, the current sensor hangs on the wire of motor driver for estimating the input electric power and torque.

After completing the setup of this experimental platform, the vibration, sound and temperature signals of the bearing, as well as the current of the motor driver can be acquired at the same time. The sampling rates of data acquisition systems are set at $2 \mathrm{MHz}$ for acoustic emission detector; $51.2 \mathrm{kHz}$ for the microphone, accelerometers and current sensor; and $1 \mathrm{~Hz}$ for thermal couple. All of the data are stored properly in cloud storage for the subsequent signal processing.

\subsection{Experimental conditions and setup}

Rotating speed of the spindle, unbalance of the shaft, and faults in the ball bearings are considered as the major input sources for simulating different operating and faults conditions. Rotating speeds used are 1000 rpm, 1500 rpm, $2000 \mathrm{rpm}$ and $2500 \mathrm{rpm}$. Fault conditions involve imbalance of the shaft, lubrication loss of bearings and lubrication contamination of bearings. There are minor and severe cases in each fault condition.

The unbalanced shaft is achieved by putting metal lumps on the disk of the shaft as shown in Fig. 3(b). The amount of imbalance is therefore the key input variable. In this study, the minor imbalance is set by $19 \mathrm{~g}$ in mass and $42 \mathrm{~mm}$ in distance, and the severe imbalance is set by $66 \mathrm{~g}$ in mass and $42 \mathrm{~mm}$ in distance.

For achieving different lubrication levels, we utilize cleaning naphtha to wash off the lubrication in the bearings to simulate the loss of lubrication, then we add grease on the bearing in different lubrication levels. Fig. 3(c-d) shows a case with fully lubrication and a case without any lubrication which can be made into other cases. In this research, the severe lack of lubrication is defined as a bearing without any lubricant, and the minor lubricated is a bearing with about $0.2 \mathrm{~g}$ lubricant.

For the issue of lubrication contamination, we utilize metal powder (Inconel 718, diameter around $50 \mu \mathrm{m}$ ) to mix with the lubricant. The proportion of lubrication and powder is $1: 0.02(\mathrm{~g})$ in minor contamination case, and 1:0.25 $(\mathrm{g})$ in severe contamination case.

In summary, there are five statuses of bearing damage used in this study. Each bearing is put on the experimental platform operating with four rotating speeds and three unbalanced conditions. Thus, there are totally 60 scenarios. In each scenario, the experiment are conducted repeatedly from stop to steady state and acquire the data to ensure the repeatability. Finally, a hundred sets of data will be collected and saved properly for each scenario.

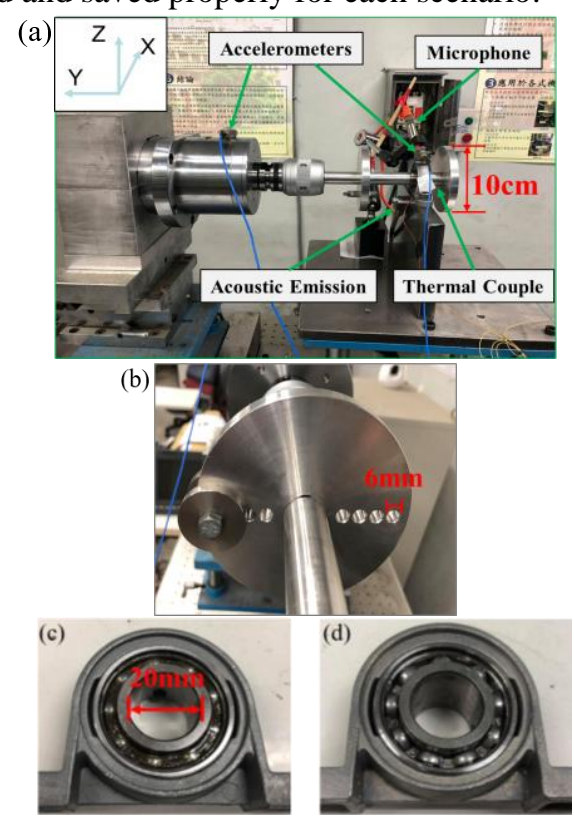

Fig. 3. Figures for (a) overall experimental platform, (b) mimic unbalance shaft by adding eccentric mass and experimental bearings (c) with fully lubrication and (d) without any lubrication. 


\section{Signal processing and analysis}

\subsection{Feature extraction}

The dimensions of original signals obtained from the experimental platform are very large. Thus, processing the data to reduce the dimensions and extract features is needed. There are many indexes that can represent the original data. Every index has different sensitivity to particular conditions. In this research, indexes can be roughly separated into two parts, namely time domain and frequency domain. First, the time-domain indexes utilized in this study and their briefly explanation are as follows.

- Root mean square (RMS): RMS is defined as the square root of the mean square. It can be applied to assess the amplitude of vibration signals.

$$
\text { Root mean square }=\sqrt{\frac{1}{n} \sum_{i=1}^{n} x_{i}^{2}}
$$

- Kurtosis: Kurtosis is defined as the fourth central moment divided by the standard deviation to the fourth power. It can be a measure of whether the data are peaked or flat.

$$
\text { Kurtosis }=\frac{1}{n} \sum_{i=1}^{n} \frac{E\left(x_{i}-\mu\right)^{4}}{\sigma^{4}}
$$

- Skewness: Skewness is defined as the third central moment divided by the standard deviation to the third power. It can be a measure of symmetry.

$$
\text { Skewness }=\frac{1}{n} \sum_{i=1}^{n} \frac{E\left(x_{i}-\mu\right)^{3}}{\sigma^{3}}
$$

- Entropy: It may represent the clutter level of the signals.

$$
\text { Entropy }=-\sum_{i=1}^{n} p\left(x_{i}\right) * \log _{2} p\left(x_{i}\right)
$$

- Crest factor: Crest factor is the peak amplitude of the signal divided by the RMS. It indicates how extreme the peaks are in a waveform.

$$
\text { Crest factor }=\frac{\left|X_{\text {peak }}\right|}{x_{r m s}}
$$

- Mean value: Mean value is an average of a signal.

$$
\text { Mean }=\frac{1}{n} \sum_{i=1}^{n} x_{i}
$$

- Standard deviation (STD): Standard deviation is a measure of the amount of variation or dispersion of a set of values.

$$
\text { Standard deviation }=\sqrt{\frac{\sum_{i=1}^{n}\left(X_{i}-\mu\right)^{2}}{n-1}}
$$

In above equations, $n$ means the number of elements in a set of signal, $x_{i}$ means the $i$-th element in the set of signal, $E$ represents the expected value, $\mu$ represents mean value, $\sigma$ represents Standard deviation, $p$ contains the normalized histogram counts in the program. All calculations of the equations are completed by MATLAB program in this work. The indexes mentioned above are utilized in time-domain signals and can be applied to different sensors. To be more specific, we use RMS, kurtosis, skewness, entropy and crest factor for the accelerometers, the microphone, and the acoustic emission detector. Moreover, we use mean value and STD for the current sensor on the motor driver.

On the other hand, the frequency-domain data as known as spectrum can be obtained by performing the Fast Fourier transform (FFT) of time-domain signal. In this study, the frequency-domain indexes are applied to the signal of the accelerometers as well as the microphone. Their briefly explanation are as follows.

- Rotating-speed-frequency magnitude: The value of the peak in the spectrum corresponding to rotating speed set at the spindle.

- Octave band induction: The summed energy of the spectrum based on octave band. In this study, we divide a spectrum into ten parts according to Table 1 and sum them up.

Table 1. Representative number of octave band

\begin{tabular}{|c|c|c|c|c|c|}
\hline No. & 1 & 2 & 3 & 4 & 5 \\
\hline Frequency & 0 & 16 & 32 & 63 & 125 \\
(Hz) & $\sim 16$ & $\sim 32$ & $\sim 63$ & $\sim 125$ & $\sim 250$ \\
\hline No. & 6 & 7 & 8 & 9 & 10 \\
\hline Frequency & 250 & 500 & 1000 & 2000 & 4000 \\
(Hz) & $\sim 500$ & $\sim 1000$ & $\sim 2000$ & $\sim 4000$ & $\sim 8000$ \\
\hline
\end{tabular}

After deciding the indexes that will be utilized. The work of signal processing can be conducted. We regarded the indexes as the features of the signals. Thus, the dimension of the signal can be well reduced and then be saved in cloud storage for subsequent utilization.

\subsection{Sensor index evaluation}

Through the effort of extracting features from original data, we can start to diagnose the status of the bearing. However, the tendency of these values vary with operating conditions. Some of the indexes may be useless in particular situations. If we make use of whole indexes to train the AI model for diagnosis, we may have a hard time dealing with the parameters in the model or obtained an unsatisfied result. Therefore, it is extremely important to perform sensor index evaluation and select proper features to train the AI model. In this approach, the accuracy of the model could be improved, and the AI technique may be used more easily. The following are three fault conditions used to demonstrate sensor index evaluation in this research.

\section{(1) Unbalance of shaft}

After completing the experiment as well as feature extraction, the database of unbalanced shaft is established. There are one hundred sets of data for each operating condition. Then we can proceed evaluation part to investigate the relationship between sensors, operating conditions and indexes. By correlating overall observations 
with domain knowledge on every feature, we can obtain the tendencies and give them a reasonable interpretation. Finally, appropriate indexes that could be the input of AI model are selected. Taking some of the indexes for example, Fig. 4(a) shows the mean results of kurtosis with respect to unbalance situations. Although the mean value may have certain correlations with the changes of unbalance level, the result is in general poor when we consider the STD of these data as shown in Fig. 4(b). Thus, the index is not proper to be used. In contrast, Fig. 5 shows the magnitude of rotating speed frequency. The value increases when the unbalance level increases, and the trend becomes more obvious when the spindle speed raises. In addition, the trend also maintains well when we consider the STD of these data. Therefore, the index is an excellent choice for training the AI model.

(a)

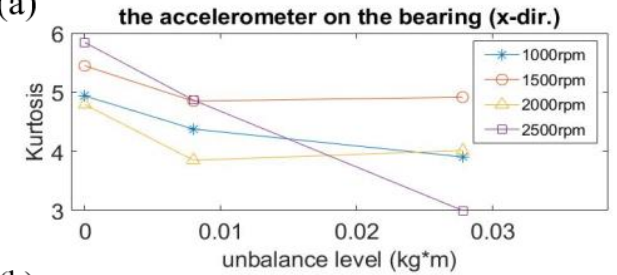

(b)

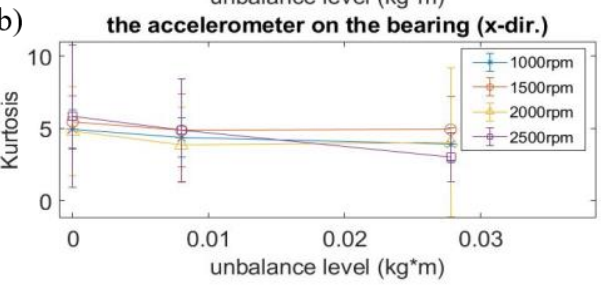

Fig. 4. Kurtosis in unbalanced conditions of the accelerometer on the bearing (a) for mean value and (b) for mean value with error bar.

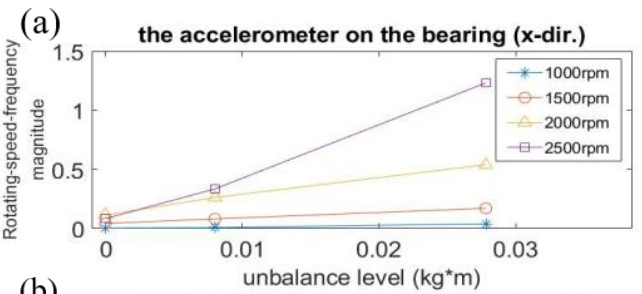

(b)

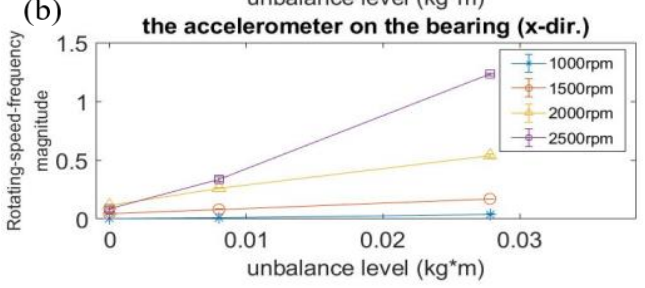

Fig. 5. Rotating-speed-frequency magnitude in unbalanced conditions for the accelerometer on the bearing (a) for mean value and (b) for mean value with error bar.

\section{(2) Loss of lubrication}

Similar to the case of shaft unbalance, we conduct overall observations with domain knowledge and take key indexes. For instance, as shown in Fig. 6(a), it indicates the correlation between the kurtosis values and the lubrication level. It also performs poor when we consider the STD of these data. On the other hand, Fig. 6(b) shows the octave band induction results of the accelerometer on the bearing. Though there is no obvious difference in low-frequency band, namely band No.1 to No.6. In band No. 8 to No.10, there is an apparent segmentation between healthy, minor and severe cases. Thus, the high-frequency bands are appropriate indexes for training the AI model.
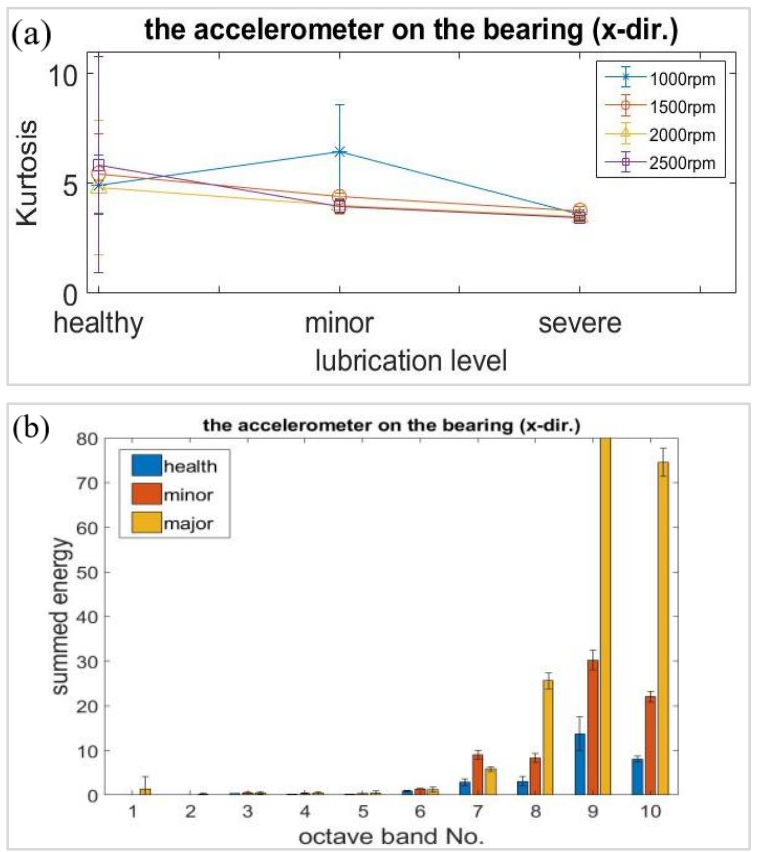

Fig. 6. Figures for (a) kurtosis values and (b) octave band induction with mean value and error bar in different lubrication levels of the accelerometer on the bearing.

\section{(3) Lubrication contamination}

Similar to operating conditions mentioned above, we perform correlation analysis to pick up key sensor indexes. As shown in Fig. 7(a), it shows that the relationship between the RMS values of accelerometer signal and the contamination levels. The values increase when the contamination levels become severe in each rotating speed. It also performs well when we consider the STD of these data. Moreover, Fig. 7(b) shows the octave band induction results of the accelerometer on the bearing. There is also an apparent segmentation between healthy, minor and severe cases in band No.8 to No.10. 

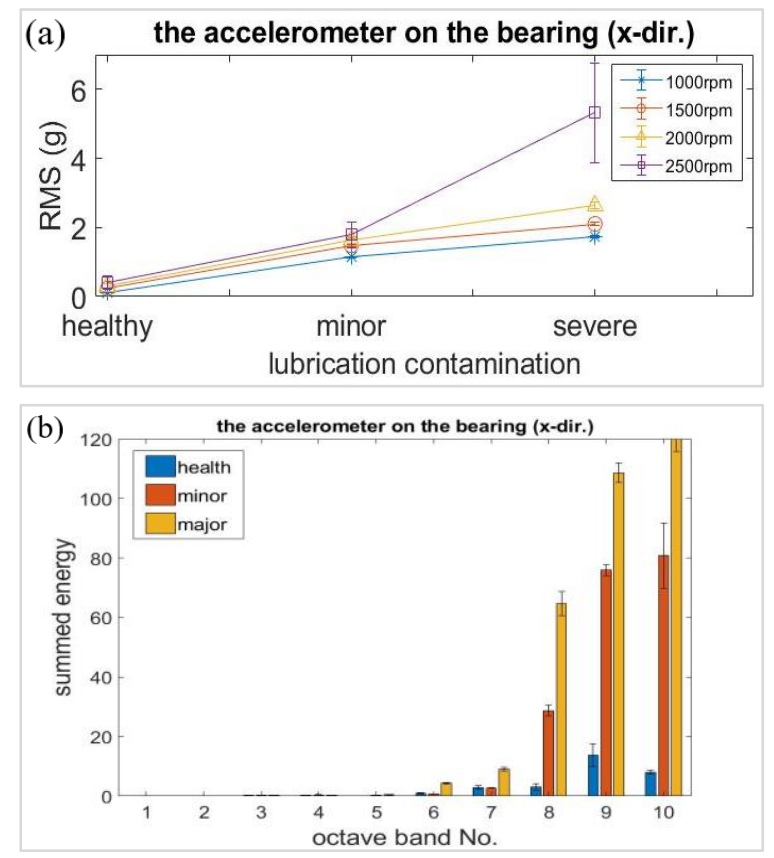

Fig. 7. Figures for (a) RMS values and (b) octave band induction with mean value and error bar in different lubrication contamination levels of the accelerometer on the bearing.

It is important to point out that we found that the dominated factor for lubrication loss and lubrication contamination are very similar. Thus, we cannot distinguish them without the aid of artificial intelligent model.

\section{Failure diagnosis using autoencoder models}

In order to establish an autoencoder model, Python, a general-purpose programming language, and PyTorch, an open source machine learning library, are utilized. In encoding process, the number of inputs is based on which faults we considered. There are two hidden layers in which the first one has 128 artificial neurons while the second one has 64. In addition, the latent space is set at three dimensions for the purpose of visualization. The optimal dimension is still unknown and is our immediate future study. Then, the decoding processing is symmetric to the encoding processing. Finally, we trained the model by 128 epochs with batch size of 64 . Thus, the dimension of features is reduced to three. We can plot the encoded features on three-dimension coordinates to make the data visible. Through data visualization, we can easily perceive the effect of trained results.

Fig. 8(a) shows the trained result for three unbalance conditions with a bearing of minor loss of lubrication at $1500 \mathrm{rpm}$. The model is trained by all indexes without any evaluation. It is obvious that the result is poor and we can hardly identify the data by its position. However, with appropriate indexes, three fault cases are clearly separated as shown in Fig. 8(b). Each case concentrates at a particular region, only few extreme values is far from the region. The model is trained by six indexes from the same dataset of which in Fig. 8(a), namely the magnitude of rotating speed frequency for the accelerometers in three axes on the bearing as well as on the spindle casing. (a)

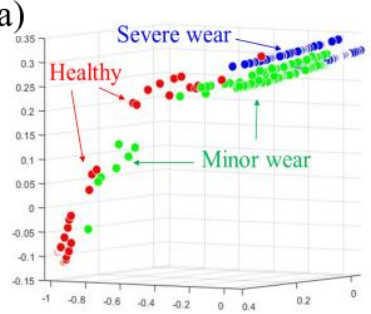

(b)

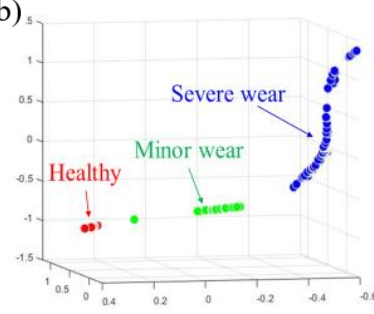

Fig. 8. Trained result using (a) all indexes and (b) proper indexes for unbalance conditions with minor loss of lubrication at $1500 \mathrm{rpm}$.

Similarly, with the same setting but change the inputs into proper indexes for diagnosing the case of lubrication loss. As shown in Fig. 9(a), the inputs of this model are RMS values as well as no. 8, 9, 10 intervals of octave band of the accelerometer attached on the bearing. The result displays that the data are mainly concentrate in three well-separated regions representing three lubrication levels can be well- classified apparently. Also, for the case of lubrication contamination, we use RMS values as well as no. 8, 9, 10 intervals of octave band induction of the accelerometer attached on the bearing. The trained result is shown in Fig. 9(b) and it also indicates a successful classification on different lubrication contamination levels. (a)

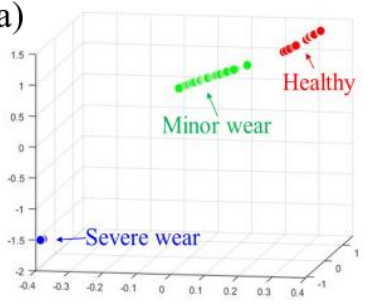

(b)

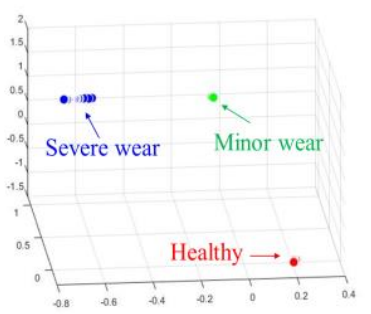

Fig. 9. Trained results using proper indexes for (a) lubrication loss and (b) lubrication contamination levels with no unbalance at $2000 \mathrm{rpm}$.

Although the severities of lubrication loss and lubrication contamination can be distinguished respectively, 
the trend and the used indexes are extremely similar. Thus, an extra autoencoder model to distinguish these two cases is required. Here we hire RMS values as well as no. 6 to 10 intervals of octave band of the accelerometer on the bearing as the inputs of this model. The result is shown in Fig. 10 and it can be seen that the healthy, severe lubrication loss, and severe lubrication contamination cases are well separated, indicated that they can be distinguished clearly.

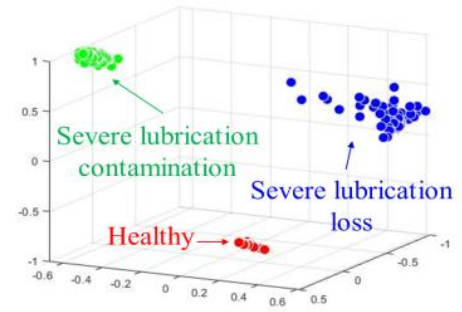

Fig. 10. Trained result using proper indexes for healthy and severe wear cases with no unbalance at $2000 \mathrm{rpm}$.

For validating the models, we input new experimental data into a trained autoencoder model. As shown in Fig. 11, the solid circle data (new data for validation) are clearly located at the same regimes as the hollow circle data (trained data). This validates the reliability of the created AI models.

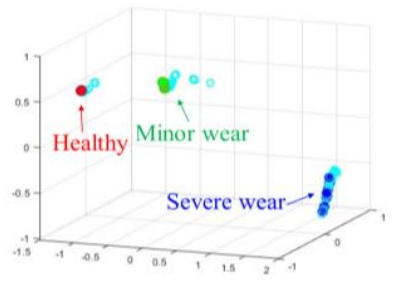

Fig. 11. Test result using proper indexes for unbalance conditions with healthy bearing at $1500 \mathrm{rpm}$.

Furthermore, the performance of the non-supervised autoencorder model may need to be quantified. Surprisingly this is not reported in previous work. Here, we define a quantitative indicator, $Q_{i j}$, as the cost function for optimization. That is,

$$
\text { Quantitative indicator }=Q_{i j}=\frac{d_{i j}}{s t d_{i}+s t d_{j}}
$$

In equation (8), $d$ means linear distance between centroids obtained by a k-means model; std means standard deviation calculated by square root of sum of squares from each axis; and $i, j$ can be 1,2 or 3 meaning healthy, minor wear and severe wear respectively. Fig. 12 shows the schematic diagram in two dimensional coordinate. In this study, three different values are calculated from each model and the minimum value is chosen as its representation.
After executing the programs a few times and comparing the quantitative indicators, the parameters of the model having the biggest value of represented quantitative indicator will be treated as the optimal parameters.

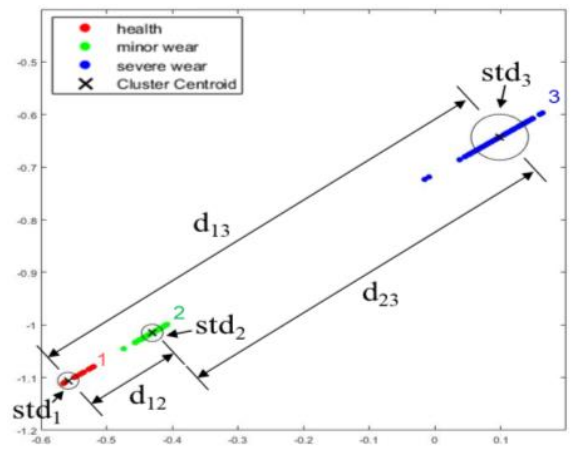

Fig. 12. Schematic diagram of quantitative indicator

Finally, it is also unknown that which dimension could have the best classification. Therefore, the autoencoder models for reducing the dimensions of each fault condition are prepared for various dimensions. Next, k-means models are utilized to obtain the labels and the cluster centroids for three groups of encoded features obtained by each autoencoder model. Each model is evaluated according to equation (8) subsequently. Thus, every optimal autoencoder model has a corresponding k-means model. Then, the models for every fault condition can be combined according to specific consequence as shown in Fig. 13 to obtain the final status diagnosis flow. Not only faults mentioned above but also other faults such as defects on outer or inner race of bearings can be added in the future.

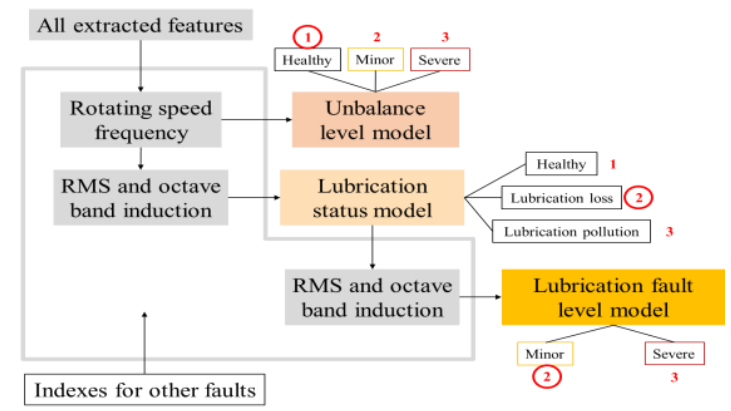

Fig. 13. Status identifying flow chart.

\section{Discussion}

An experimental platform with multiple sensors is set up in this research. Five bearings with different lubrication conditions are operated under three unbalance conditions at four rotating speeds. The raw signals are saved to conduct signal processing. Then, comprehensive observations for the relationship between sensors, operating conditions and 
indexes is carried out. First, in aspect of unbalance, the rotating-speed-frequency magnitudes of accelerometers in three axes on both bearing and spindle casing are selected through sensor index evaluation. Although the trends of each axis may change, the overall trend is sensitive to operating parameters. In addition, the trend is also suitable for different status of bearings and becomes more apparent with the increase of rotating speed. Second, in aspect of lubrication loss, the RMS as well as no. 8, 9 and 10 octave band of the accelerometer on bearing are selected. The trend also becomes more obvious with the increase of rotating speed. Third, in aspect of lubrication contamination, the RMS as well as no. 8, 9 and 10 octave band of the accelerometer on bearing are selected. The trend is similar to the case of lubrication loss, but the minor difference can be carried out with the aid of AI model. All faults can be identified with specific consequence. Overall, the trained results of AI models perform well with selected indexes.

Though some indexes seem useless in this study, it may become useful when other faults are considered. In terms of AI model, it can represent excellent results to the situation we have discussed. When the unknown failure appears, we can find out the situation and discuss individually to make the diagnosis system more complete.

\section{Conclusion}

This study represents a preliminary achievement of status diagnosis for ball bearings failure using autoencoder and k-means models to help users identify the faults by specific consequence. The concept can be promoted to other components of machines. By sensor index evaluation, sensitive features can be chosen to train the AI model. Through the efforts of evaluation, the accuracy of the AI model can be improved. Also, the difficulty of modifying the parameters in AI model is reduced significantly. The diagnosis method can be utilized to smart factory naturally in the future. Finally, this research is not completed yet and more bearing faults that commonly appear in factories should be discussed to make this method more reliable.

\section{Acknowledgment}

This work is supported by the ministry of science and technology (MOST) of Taiwan under contract numbers MOST 109-2221-E-006-096, MOST 109-2622-8-006-005 and MOST 109-2221-E-218-007. In addition, the support from Syntec Technology Company is greatly appreciated.

\section{References}

(1) Y. Lu, "Industry 4.0: A Survey on Technologies, Applications and Open Research Issues," Journal of Industrial Information Integration, vol. 6, pp. 1-10, Jun. 2017.

(2) C. Scheffer and P. Girdhar, Practical Machinery Vibration Analysis \& Predictive Maintenance. Amsterdam, The Netherlands: Elsevier, 2004.

(3) D. Yu, J. S. Cheng, and Y. Yu, "Application of EMD method and Hilbert spectrum to the fault diagnosis of roller bearings," Mechanical Systems and Signal Processing, vol.19, no.2, pp. 259-270, Mar. 2005.

(4) P. K. Kankar, S. C. Sharma, and S. P. Harsha, "Fault diagnosis of ball bearings using continuous wavelet transform," Applied Soft Computing, vol. 11, no.2, pp. 2300-2312, Mar. 2011.

(5) R. B. Randall, "Detection and diagnosis of incipient bearing failure in helicopter gearboxes," Engineering Failure Analysis, vol. 11, no.2, pp. 177-190, Apr. 2004.

(6) F. Elasha, M. Greaves, D. Mba, and A. Addali, "Application of Acoustic Emission in Diagnostic of Bearing Faults within a Helicopter gearbox," Procedia CIRP, vol. 38, pp. 30-36, 2015.

(7) F. Q. Lauzon, "An introduction to deep learning," The 11th International Conference on Information Sciences, Signal Processing and their Applications, Montreal, Canada, 2012, pp.1438-1439.

(8) B. Li, M. Y. Chow, Y. Tipsuwan, and J. C. Hung, "Neural-network-based motor rolling bearing fault diagnosis," IEEE transactions on industrial electronics, vol. 47, no.5, pp. 1060-1069, Oct. 2000.

(9) P.K. Kankar, S. C. Sharma, and S.P. Harsha, "Fault diagnosis of ball bearings using machine learning methods," Expert Systems with Applications, vol. 38, no.3, pp. 1876-1886, Mar. 2011.

(10)W. Wang, Y. Huang, Y. Wang, and L. Wang, "Generalized Autoencoder: A Neural Network Framework for Dimensionality Reduction," 2014 IEEE Conference on Computer Vision and Pattern Recognition Workshops, Columbus, OH, 2014, pp. 496-503.

(11) K. G. Lore, A. Akintayo, and S. Sarkar, "LLNet: A deep autoencoder approach to natural low-light image enhancement," Pattern Recognition, vol. 61, pp. 650-662, Jan. 2017.

(12) M. Yousefi-Azar, V. Varadharajan, L. Hamey, and U. Tupakula, "Autoencoder-based feature learning for cyber security applications," 2017 International Joint Conference on Neural Networks (IJCNN), Anchorage, AK, 2017, pp. 3854-3861.

(13) H. Shao, H. Jiang, H. Zhao, and F. Wang, "A novel deep autoencoder feature learning method for rotating machinery fault diagnosis," Mechanical Systems and Signal Processing, vol. 95, pp. 187-204, Oct. 2017.

(14) J. Wu, H. Xiong, and J. Chen, "Adapting the right measures for k-means clustering," Proceedings of the 15th ACM SIGKDD international conference on knowledge discovery and data mining, Paris, France, 2009, pp. 877-885 